\title{
Memory guidance in distractor suppression is governed by the availability of cognitive control
}

\author{
Wen Wen ${ }^{1,2,3,4} \cdot$ Yin $\mathrm{Hou}^{5} \cdot$ Sheng $\mathrm{Li}^{1,2,3,4}$ \\ Published online: 26 March 2018 \\ (C) The Psychonomic Society, Inc. 2018
}

\begin{abstract}
Information stored in the memory systems can affect visual search. Previous studies have shown that holding the to-be-ignored features of distractors in working memory (WM) could accelerate target selection. However, such facilitation effect was only observed when the cued to-be-ignored features remained unchanged within an experimental block (i.e., the fixed cue condition). No search benefit was obtained if the to-be-ignored features varied from trial to trial (i.e., the varied cue condition). In the present study, we conducted three behavioral experiments to investigate whether the WM and long-term memory (LTM) representations of the to-be-ignored features could facilitate visual search in the fixed cue (Experiment 1) and varied cue (Experiments 2 and 3 ) conditions. Given the importance of the processing time of cognitive control in distractor suppression, we divided visual search trials into five quintiles based on their reaction times (RTs) and examined the temporal characteristics of the suppression effect. Results showed that both the WM and LTM representations of the to-be-ignored features could facilitate distractor suppression in the fixed cue condition, and the facilitation effects were evident across the quintiles in the RT distribution. However, in the varied cue condition, the RT benefits of the WM-matched distractors occurred only in the trials with the longest RTs, whereas no advantage of the LTM-matched distractors was observed. These results suggest that the effective WM-guided distractor suppression depends on the availability of cognitive control and the LTM-guided suppression occurs only if sufficient WM resource is accessible by LTM reactivation.
\end{abstract}

Keywords Attention and memory $\cdot$ Cognitive and attentional control $\cdot$ Visual search

\section{Introduction}

Searching for a visual target in a dense forest or a busy city is very challenging. A large number of distractors often put our limited attentional capability to the test. Nevertheless, searching

Sheng Li

sli@pku.edu.cn

1 School of Psychological and Cognitive Sciences, Peking University, 5 Yiheyuan Road, Haidian, Beijing 100871, China

2 Beijing Key Laboratory of Behavior and Mental Health, Peking University, Beijing 100871, China

3 PKU-IDG/McGovern Institute for Brain Research, Peking University, Beijing 100871, China

4 Key Laboratory of Machine Perception (Ministry of Education), Peking University, Beijing 100871, China

5 Department of Psychology, School of Sociology and Psychology, Central University of Finance and Economics, Beijing 100081, China difficulty could be greatly reduced if the critical features of the target are foreknown and stored in working memory (WM) as search templates (Bundesen, 1990; Desimone \& Duncan, 1995; Wolfe, 1994). Sometimes, apart from information about the target, we could also obtain information about the to-be-ignored distractors. This raises the question that whether we could avoid paying attention to these distractors during the search. Previous studies have demonstrated that WM-matched distractors attract attention even when the participants were informed that those distractors would never be the target (Downing, 2000; Hollingworth \& Beck, 2016; Mannan, Kennard, Potter, Pan \& Soto, 2010; Olivers, Meijer, \& Theeuwes, 2006; Soto, Heinke, Humphreys, \& Blanco, 2005; Soto \& Humphreys, 2007, 2009; for a review, see Soto, Hodsoll, Rotshtein, \& Humphreys, 2008). According to these findings, top-down control may not be able to override the involuntary capture by WM-matched distractors.

Nonetheless, another line of research has provided evidence that was inconsistent with the uncontrollable nature of attentional capture by WM-matched distractors. These results have demonstrated that simply holding an item in WM was not sufficient for 
biasing attention toward the perceptual inputs that matched its critical feature (Dalvit \& Eimer, 2011; Carlisle \& Woodman, 2011; Houtkamp \& Roelfsema, 2006; Peters, Goebel, \& Roelfsema, 2009; Olivers, 2009; Lu et al., 2017; for a review, see Olivers, Peters, Houtkamp, \& Roelfsema, 2011). Furthermore, several studies have shown that WM representations could be used to inversely guide attention away from the WM-matched distractors (Arita, Carlisle, \& Woodman, 2012; Cunningham \& Egeth, 2016; Downing \& Dodds, 2004; Han \& Kim, 2009; Woodman \& Luck, 2007) and this effect was facilitated by the rewarding history of the WM representations (Gong, Yang, \& Li, 2016). These studies argued that the deployment of attention to the WM-matched items could be strategically controlled to meet task requirements (Geng, 2014; Kiyonaga, Egner, \& Soto, 2012; Woodman, Carlisle, \& Reinhart, 2013). Therefore, two distinct phenomena concerning the WM guidance on attention have been suggested. Attention could either be captured by or guided away from the WM-matched distractors, resulting in opposite behavioral consequences. Identifying the critical factors that could explain the controversy about the effects of WM guidance in attention control is a necessary step towards further understanding of WM-attention interaction.

In most studies that observed successful suppression of WM-matched distractors, the cue that indicated the to-be-ignored features in visual search remained unchanged within a block of trials (i.e., the fixed cue condition) (Cunningham \& Egeth, 2016; Gaspelin, Leonard, \& Luck, 2015, 2017; Moher, Lakshmanan, Egeth, \& Ewen, 2014; Noonan et al., 2016; Vatterott \& Vecera, 2012). Of particular relevance to the present study, Cunningham and Egeth (2016) have found that participants could use the cued to-be-ignored features to facilitate their search only in the fixed cue condition as compared with the trials with a neutral cue. However, there was no reaction time (RT) benefit when the cued to-be-ignored features varied from trial to trial (i.e., the varied cue condition). They attributed the absence of facilitation effect in the varied cue condition to the failure of forming a stable representation of the to-be-ignored feature in WM named as rejection template (Arita et al., 2012; Cunningham \& Egeth, 2016; Woodman \& Luck, 2007). Meanwhile, utilizing such rejection template requires the involvement of cognitive control (Han \& Kim, 2009). Hence, cognitive control could be a critical factor that accounts for the discrepancy between the fixed cue and varied cue conditions, because of its involvement in both the creation and execution of the rejection template. In the varied cue condition where WM representations are frequently switched between trials, suppression benefits might occur when the actual response time is long enough for the completion of effective cognitive control. Thus, in the present study, our first aim was to elucidate the influence of the available cognitive control on distractor suppression in the varied cue condition.

Meanwhile, recent studies have suggested that multiple memory systems that include WM and LTM contribute to attentional control (Hutchinson \& Turk-Browne, 2012; Woodman \& Chun, 2006). This proposal was supported by experimental investigations in which the content of LTM has been shown to optimize perceptual sensitivity (Stokes, Atherton, Patai, \& Nobre, 2012) and induce attentional capture (Fan \& Turk-Browne, 2016; Olivers, 2011). Nevertheless, the interaction between the predictive information in LTM and distractor suppression remained less explored. Recently, Gong and colleagues have shown that previously learned reward-feature associations could facilitate distractor suppression when the reward-associated feature always served as physically salient distractor (Gong, Jia, \& Li, 2017). Despite not being designed to directly test the modulation effect of the LTM representations in distractor suppression, this study has suggested the potential benefits of the previously learned distractor information in visual search. Therefore, the second issue we aimed to address was whether and under what specific circumstances the LTM information could facilitate distractor suppression. In the context of cued visual search in the present study, LTM information referred to information that was provided at the beginning of the experiment and was not actively cued on every trial.

We conducted three behavioral experiments to address these two issues. In Experiment 1, we replicated the findings of previous literature by demonstrating the RT benefits of fixed cue in distractor suppression. We also showed that the LTM representation of a constant color that predicted the occasional appearance of its associated distractors induced similar RT benefits in the fixed cue condition. In Experiments 2 and 3, we examined the RT benefits of the WM and LTM representations for distractor suppression in the varied cue condition. These two experiments used the same and a different set of visual stimuli as in Experiment 1, respectively. The results from the two experiments agreed with each other and showed that the RT benefits in the varied cue condition could only be observed in the trials with the longest RTs, whereas there were no RT benefits of the LTM-held constant color in the varied cue condition. Taken together, these results suggested that the availability of cognitive control was critical for memory-guided distractor suppression in both the fixed cue and varied cue conditions.

\section{Experiment 1}

Experiment 1 was designed to examine the RT benefits of distractor suppression from the WM and LTM representations in the fixed cue condition. We adopted a dual-task paradigm where a to-be-memorized color cue appeared before search array and indicated that the target would not share the same color with the cue. If participants could strategically develop a rejection template using the color cue, they should be able to avoid unnecessary capture by the distractors in the cued color. We also designated a constant color that occasionally appeared in the search display but was never associated with 
the target. We aimed to test whether prior information of distractors' color in LTM could be used to facilitate visual search in the context of fixed cue.

\section{Method}

\section{Participants}

Twenty-five naïve participants (11 males; age range: 19-26 years; mean age: 22.4 years; all right-handed) participated in the experiment and were paid for their participation. All of the participants had normal or corrected-to-normal visual acuity and normal color vision, none of them had known neurological or visual disorders. We used G-power (Faul, Erdfelder, Lang, \& Buchner, 2007), given an fof 0.25 (which represents medium effect size) and power of $80 \%$, to estimate how many participants should be collected for the experiment (the same procedure was applied to Experiments 2 and 3). Although the expected sample size was 28 , we stopped data collection after 25 participants. This was mainly due to the similar sample size in previous related studies (Cunningham \& Egeth, 2016; Han \& Kim, 2009) and the consistent behavioral pattern across participants. However, we strictly followed the suggested sample size (i.e., 28) in Experiments 2 and 3. Participants provided written informed consent before the experiment. The study was approved by the local ethics committee.

\section{Stimuli and apparatus}

The experiments were programmed with Psychtoolbox 3 (Brainard, 1997; Kleiner et al., 2007) in a MATLAB (Mathworks, Natick, MA, USA) programming environment. The stimuli were displayed on a CRT monitor with a refresh rate of $100 \mathrm{~Hz}$ and a resolution of $1,024 \times 768$ pixels. Participants sat at a distance of $70 \mathrm{~cm}$ to the screen with a chin-rest to stabilize the head position. Stimuli were selected from a set of seven equiluminous CIELAB colors (physical luminance of the seven colors ranged from $28.5 \mathrm{~cd} / \mathrm{m}^{2}$ to $32.2 \mathrm{~cd} / \mathrm{m}^{2}$, white background was $78.4 \mathrm{~cd} / \mathrm{m}^{2}$ ) and there were three shapes in the search array (circle: $1.4^{\circ} \times 1.4^{\circ}$; diamond: $1.6^{\circ} \times 1.6^{\circ}$; hexagon: $1.4^{\circ} \times 1.4^{\circ}$ ).

\section{Procedure}

We modified the paradigm of Gaspelin et al. $(2015,2017)$ by adding an initial color cue and a retention period. As shown in Fig. 1a, each trial began with the presentation of a central black fixation cross for $500 \mathrm{~ms}$. Before the onset of the search array, a color cue $\left(0.4^{\circ} \times 0.4^{\circ}\right)$ was presented for $500 \mathrm{~ms}$, indicating that the target was not in the cued color in the following search array. Color cue remained the same within one block but changed across blocks. After a 1-s blank, the search array appeared for $150 \mathrm{~ms}$ before being masked by six grayscale checkerboard squares $\left(1.6^{\circ} \times 1.6^{\circ}\right)$. The mask remained visible until the participants' response or $3 \mathrm{~s}$ elapsed. The search array was composed of six shapes forming an imaginary circle with a radius of $2^{\circ}$. Search target was a randomly chosen unique shape that was explicitly informed to each participant and was the same across blocks. The shape of the target was counterbalanced across participants. The six shapes were in two different colors: the target and two of the distractors were in the target's color and the remaining three distractors were in the distractor color. Specifically, the distractor color was defined as the color that was different from the target's color (note that two out of five distractors were in the same color as the target). Inside of each shape, there was a black dot $\left(0.2^{\circ} \times 0.2^{\circ}\right)$ located either at the left or right side $\left(0.2^{\circ}\right.$ to the edge).

There were two types of trials. In $90 \%$ of the trials (the search trials), participants were instructed to indicate the location of the dot inside the target shape by pressing left or right arrow key using their right hands. In $10 \%$ of the trials (the probe trials), after a $150 \mathrm{~ms}$ presentation of the search array, six randomly chosen capital letters ( $0.8^{\circ}$ in height) superimposed on each shape for $100 \mathrm{~ms}$ and then were masked by six checkerboard squares for $500 \mathrm{~ms}$. During the recall phase, participants were given unlimited amount of time to choose all the remembered letters from an onscreen alphabet using the mouse. At the end of the search and probe trials, the participants were required to complete a change detection task by judging whether the presented color square $\left(0.4^{\circ} \times 0.4^{\circ}\right)$ was in the same color as the cued color in the same trial by pressing $\mathrm{Y}$ or $\mathrm{N}$ using their left hand within $3 \mathrm{~s}$. The change detection task was the same for all three conditions. The inter-trial interval was set to $1.5 \mathrm{~s}$.

The trials in each type were classified into three (WM-matched, LTM-matched, and neutral) conditions based on the cueing contingency of the distractor color (Fig. 1B). In the WM-matched condition ( $60 \%$ of the trials), the distractor color was the cued color. We deliberately assigned a larger proportion of the trials to this condition to encourage the participants to use the color cue for later visual search. In the LTM-matched condition ( $20 \%$ of the trials), the distractor color was a constant color (green). The participants were informed at the beginning of the experiment that the target would never be in this constant color. In the neutral condition (20\% of the trials), the distractor color was a randomly selected color that was neither the cued color nor the constant color. For all three conditions, the target's color was a randomly selected color from color set by excluding the cued color and the constant color.

There were six blocks and each contained 100 trials with three conditions (WM-matched, LTM-matched, and neutral) and two trial types (search and probe) randomly mixed according to the pre-defined proportions. Each block began with a 10 trials practice which was excluded from the analysis. Participants were encouraged to respond as quickly as possible without risking accuracy. 


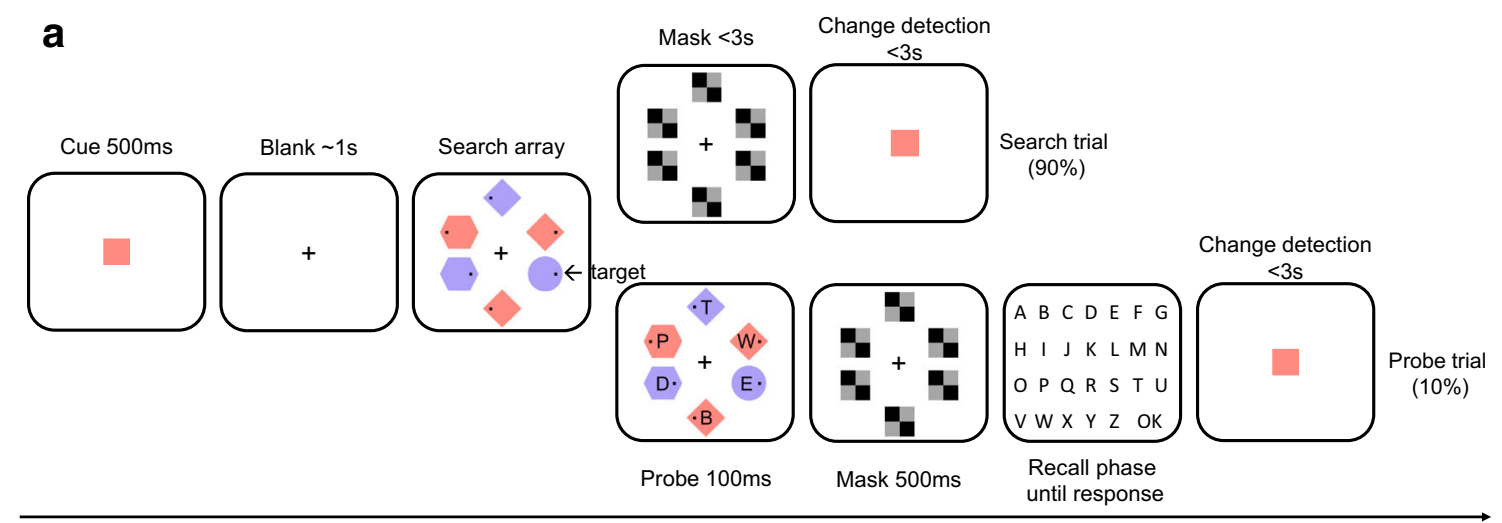

b

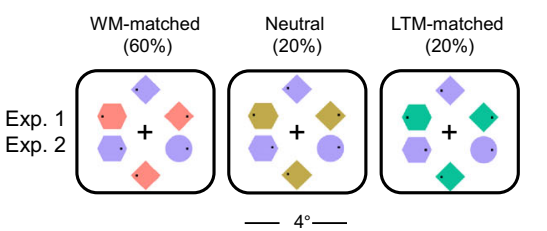

Cueing contingency

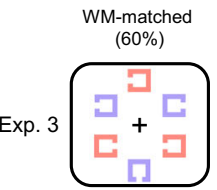
Fig. 1 Schematic illustrations of the experimental procedure and the
cueing contingencies of the distractor color. (a) An example of working memory (WM)-matched trial from Experiments 1 or 2. The color cue at the beginning of a trial indicated that items in this color would never be the target in the search array. The presentation time of search array in Experiment 1 was $150 \mathrm{~ms}$ while it was titrated for each participant in Experiments 2 and 3. In search trials, participants were instructed to discriminate the dot's location inside the target shape (circle in this example) in Experiments 1 and 2 or the gap's location of the target

\section{Data analysis}

We only analyzed the search trials with correct responses in both visual search task and change detection task. This resulted in $18.06 \%$ of the search trials being dropped. For the rest of the trials, for each condition in each block, we excluded responses faster than $200 \mathrm{~ms}$ and recursively removed the trials with RTs less than and beyond 2.5 standard deviation from the mean (Van Selst \& Jolicoeur, 1994). This resulted in 5.90\% and $4.33 \%$ of all search trials removed based on search RT and change detection RT, respectively. All probe trials were involved for letter recall analysis. For all statistical analyses, we adopted Greenhouse-Geisser correction in case of sphericity violations and Boferroni correction for multiple comparison. The comparisons of interest were WM-matched and LTM-matched conditions versus neutral condition.

\section{Results}

\section{Search trials}

As shown in Fig. 2a, one-way repeated measures ANOVA on search RTs yielded a significant main effect among the three cueing contingencies $\left(F(2,48)=10.572, p<.001, \eta^{2}=\right.$ $0.306)$. Search RTs in the WM-matched $(p=.008)$ and
C Distractor locations
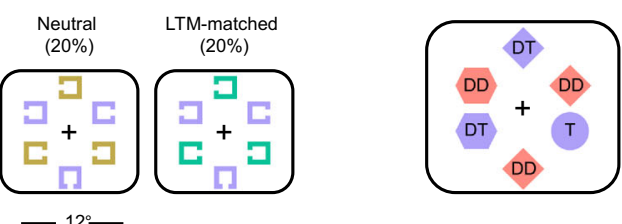

Landolt-square (with an upward or downward gap) in Experiment 3. Participants performed change detection in both the search and probe trials by indicating whether the presented color was the same as the cued color. The color cue, which remained unchanged within a block in Experiment 1 (the fixed cue condition), changed every trial in Experiments 2 and 3 (the varied cue condition). There were no probe trials in Experiment 3. (b) Search arrays of three cueing contingencies. (c) Three types of distractors position. $D T$ distractors in target color, $D D$ distractors in distractor color

LTM-matched $(p<.001)$ conditions were significantly faster than those in the neutral condition. These results had two implications. First, the results agreed with the previous findings that fixed to-be-ignored cues held in WM can accelerate distractor suppression (Cunningham \& Egeth, 2016; Noonan et al., 2016). Second, the to-be-ignored feature held in LTM can be effectively applied in distractor suppression when its associated distractors occasionally appeared in the context of the fixed cue condition.

In order to investigate the role of cognitive control in distractor suppression, we examined the possible relationship between the time available for cognitive control to take place and the observed suppression effect. For each participant, we merged the data of all six blocks and then, for each cueing contingency, grouped trials into five discrete bins based on quintiles of search RT distribution (i.e., 20, 40, 60, 80, and 100 percentiles) (Fig. 2b). Two-way repeated measures ANOVA on RT quintile and cueing contingency revealed significant main effects on RT quintile $(F(4,96)=192.805, p<$ $\left..001, \eta^{2}=0.889\right)$, cueing contingency $(F(2,48)=10.415, p$ $\left.<.001, \eta^{2}=0.303\right)$, and their interaction $(F(8,192)=4.023, p$ $\left.=.016, \eta^{2}=0.144\right)$. Simple effect analysis revealed that three conditions differed under all quintiles (all $p s<.05$ ). The RT benefits in the WM-matched and LTM-matched conditions were observed even in those trials with the shortest search RTs. 

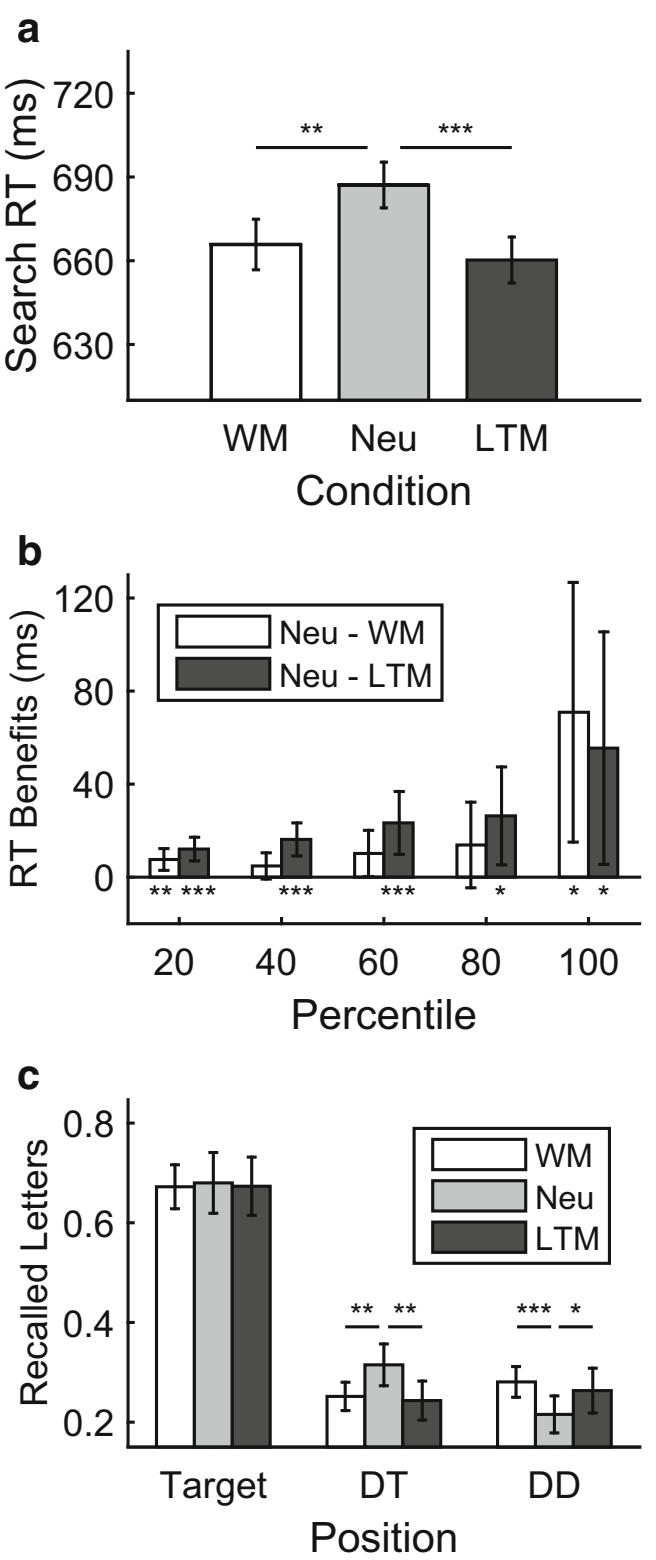

Fig. 2 Results of Experiment 1. (a) Search RTs of the three cueing contingency conditions in the search trials. (b) RT benefits in five RT percentiles as measured by subtracting the RTs in the neutral condition from the WM-matched and LTM-matched conditions in the search trials. (c) Number of correctly recalled letters per location of each type (target, DT, and DD) in the probe trials. For each type of position, the value is the total recalled letters from all locations of this type divided by its location number ( 1 for target, 2 for DT, and 3 for DD). Error bars represent withinsubject $95 \%$ confidence interval. Asterisks represent significances $(*$ $p<.05, * * p<.01, * * * p<.001) . D T$ distractors in target color, $D D$ distractors in distractor color

Change detection RTs also differed among the three cueing contingencies $\left(F(2,48)=7.587, p=.001, \eta^{2}=.240\right)$. Change detection RTs in the WM-matched condition was faster than that in the neutral $(p<.001)$ and LTM-matched $(p=.049)$ conditions while no significant difference was observed between the latter two $(p=.869)$. These results could be resulted from the appearance of another color (the LTM-held constant color or the neutral color) in the search array that impaired the WM representation of the WM cue and therefore led to a slowdown in detection performance (Kiyonaga et al., 2012). As for search accuracy and change detection accuracy, there were no significant differences among the three conditions (search accuracy: $F(2,48)=1.649, p=.203$; change detection accuracy: $F(2,48)=2.116, p=.132$, descriptive statistics were provided in Table 1).

\section{Probe trials}

Participants correctly recalled 1.98 letters per trial on average and there was no difference among three cueing contingencies $(F(2,48)=1.549, p=.223$, Table 2$)$. The six to-be-recalled letters were grouped into three types of position, according to the color and shape of the search items where they were embedded (Fig. 1c): the target, the two distractors in target color (abbr. DT), and the three distractors in distractor color (abbr. DD). The number of the correctly recalled letters at each letter position for each cueing contingency was shown in Fig. 2c. Letter position (target, DT, and DD) and cueing contingency (WM-matched, LTM-matched, and neutral) were submitted to a repeated measures ANOVA. There was a significant main effect of letter position $\left(F(2,48)=190.802, p<.001, \eta^{2}=\right.$ .888 ) but not of cueing contingency $(F(2,48)=0.569, p=$ $.570)$. Crucially, there was a significant interaction effect $(F$ $\left.(4,96)=4.103, p=.011, \eta^{2}=.146\right)$. The three cueing contingencies had equal number of recalled letters at the position of the target $(F(2,48)=.04, p=.963)$, but not at the position of DT $\left(F(2,48)=7.85, p<.001, \eta^{2}=.258\right)$ or distractor color $(F$ $\left.(2,48)=9.63, p<.001, \eta^{2}=.300\right)$. At the position of DT, participants recalled more letters in the neutral condition as compared with the WM-matched $(p=.010)$ and LTM-matched $(p=.009)$ conditions. At the position of DD, the result pattern was reversed: participants recalled more letters in the WM-matched $(p<.001)$ and LTM-matched $(p=.043)$ conditions as compared with the neutral condition. There were no differences among three cueing contingencies for the accuracy and RTs of the change detection task $(F(2,48)=0.264, p=$ $.715, F(2,48)=1.687, p=.196$, respectively $)$.

\section{Discussion}

The results of the search trials demonstrated that participants could effectively suppress the distractors that matched the WM representations of the to-be-ignored cues in the fixed cue condition. Meanwhile, the RT benefits obtained in the LTM-matched condition indicated that the LTM information could also guide attention away from the matched distractors under the context of fixed distractor cues. In contrast to Gaspelin et al. (2015), our participants recalled more letters at the positions of DD in WM-matched and LTM-matched than in neutral condition. One plausible explanation for this 
Table 1 Mean RTs and accuracies (with standard deviation) for the search and probe trials in the three experiments

\begin{tabular}{|c|c|c|c|c|c|c|c|}
\hline & & \multicolumn{4}{|l|}{ Search trials } & \multicolumn{2}{|l|}{ Probe trials } \\
\hline & & Search RT & Search ACC & $\begin{array}{l}\text { Change } \\
\text { detection RT }\end{array}$ & $\begin{array}{l}\text { Change } \\
\text { detection ACC }\end{array}$ & $\begin{array}{l}\text { Change } \\
\text { detection RT }\end{array}$ & $\begin{array}{l}\text { Change } \\
\text { detection ACC }\end{array}$ \\
\hline \multirow{3}{*}{ Experiment 1} & WM & $666(83)$ & $0.83(0.09)$ & $626(105)$ & $0.98(0.02)$ & $1074(217)$ & $0.96(0.04)$ \\
\hline & Neutral & $687(85)$ & $0.85(0.08)$ & 648 (110) & $0.97(0.02)$ & $1122(230)$ & $0.97(0.05)$ \\
\hline & LTM & $660(87)$ & $0.84(0.09)$ & $641(102)$ & $0.97(0.02)$ & $1083(222)$ & $0.96(0.08)$ \\
\hline \multirow[t]{3}{*}{ Experiment 2} & WM & $690(115)$ & $0.77(0.06)$ & $541(77)$ & $0.94(0.04)$ & 935 (217) & $0.79(0.11)$ \\
\hline & Neutral & 708 (127) & $0.76(0.06)$ & $575(85)$ & $0.91(0.06)$ & 977 (224) & $0.65(0.18)$ \\
\hline & LTM & $703(120)$ & $0.77(0.05)$ & 588 (107) & $0.90(0.07)$ & $973(251)$ & $0.68(0.17)$ \\
\hline \multirow[t]{3}{*}{ Experiment 3} & WM & $813(153)$ & $0.74(0.05)$ & $532(90)$ & $0.95(0.04)$ & & \\
\hline & Neutral & $822(170)$ & $0.74(0.06)$ & $543(93)$ & $0.91(0.07)$ & & \\
\hline & LTM & $828(165)$ & $0.73(0.06)$ & $570(115)$ & $0.92(0.08)$ & & \\
\hline
\end{tabular}

contradiction was that participants reallocated their attention when the letters appeared signaling the switch to a probe trial. During the reallocation process, stimuli matched memory representation would have a competitive advantage over those neutral stimuli and resulted in the capture effect in the probe trial.

\section{Experiment 2}

The goal of Experiment 2 was to investigate whether participants could create a rejection template based on varied WM representations and use it to facilitate distractor suppression.

\section{Method}

\section{Participants}

Twenty-nine new participants (10 males; age range: 18-28 years; mean age: 22.3 years; all right-handed) participated in the experiment and were paid for their participation. All participants had normal or corrected-to-normal visual acuity and

Table 2 Mean number (with standard deviation) of correctly recalled letters in the probe trials in Experiments 1 and 2

\begin{tabular}{lllll}
\hline & & Target & $\begin{array}{l}\text { Distractor in } \\
\text { target color } \\
\text { (DT) }\end{array}$ & $\begin{array}{l}\text { Distractor in } \\
\text { distractor } \\
\text { color (DD) }\end{array}$ \\
\hline Experiment 1 & WM & $0.67(0.15)$ & $0.25(0.09)$ & $0.28(0.11)$ \\
& Neutral & $0.68(0.19)$ & $0.32(0.12)$ & $0.22(0.11)$ \\
& LTM & $0.67(0.19)$ & $0.24(0.11)$ & $0.26(0.11)$ \\
Experiment 2 & WM & $0.47(0.16)$ & $0.20(0.11)$ & $0.20(0.11)$ \\
& Neutral & $0.46(0.18)$ & $0.21(0.12)$ & $0.20(0.11)$ \\
& LTM & $0.50(0.21)$ & $0.24(0.15)$ & $0.18(0.12)$ \\
\hline
\end{tabular}

normal color vision. None of them had known neurological or visual disorders. One subject was excluded from the analysis because of extremely poor performance in one block (no search trials that both the search and change detection tasks were correct).

\section{Stimuli and apparatus}

The stimuli and apparatus were the same as in Experiment 1.

\section{Procedure}

The main procedure in Experiment 2 was the same as in Experiment 1 with the following five exceptions. First, the cued color changed randomly from trial to trial. Second, an articulatory suppression task was added to prevent verbal rehearsal during WM maintenance (Downing \& Dodds, 2004; Houtkamp \& Roelfsema, 2006; Woodman \& Luck, 2007). Three randomly selected digits were shown on the screen for $500 \mathrm{~ms}$ at the beginning of each trial and the participants were required to verbally repeat them throughout the whole trial. Third, the constant color that served as LTM representation was counterbalanced across participants to reduce personal color preference. Fourth, a 1-up/2-down staircase session was conducted to obtain an appropriate presentation duration of the search array for each participant. We found a strong practice effect in Experiment 1 as indicated by the increased search accuracy across six blocks $(F(5,120)=7.716, p<$ $.001, \eta^{2}=.243$ ). Participants who had sufficient time to fulfill search task might strategically refresh WM representation by attending to the WM matched distractors to achieve better change detection performance (Woodman \& Luck, 2007; Yi, Turk-Browne, Chun, \& Johnson, 2008), leading to a prolonged search RT. The staircase procedure was introduced to control for this possibility. Participants came twice on two consecutive days and the staircase session was performed on 
the first day. Only the participants with a threshold around 100 200 ms continued their participation in the main experiment on the second day. The mean threshold across the participants was $137 \mathrm{~ms}$. Fifth, we emphasized the hit rate in the probe trials because the guess rate $(39 \%)$ was relatively high in Experiment 1.

\section{Data analysis}

The procedures for data exclusion and statistical analysis were the same as in Experiment 1, resulting in $28.4 \%$ of the trials being dropped for incorrect performance in either the search or change detection tasks. In addition, $4.81 \%$ and $4.72 \%$ of all search trials were excluded because of the out of RT range in the search and change detection tasks respectively. No probe trial was excluded from the analysis.

\section{Results}

\section{Search trials}

We did not observe significant differences across the three cueing contingencies in either search RTs $(F(2,54)=1.845$, $p=.168$, Fig. 3a) or search accuracy $(F(2,54)=0.936, p=$ .398). As in Experiment 1, we compared the three cueing contingencies in five RT quintiles (Fig. 3b). There was a significant main effect on quintiles $(F(4,108)=149.413, p<$ $\left..001, \eta^{2}=0.847\right)$, but the main effect on cueing contingency was not significant $(F(2,54)=1.876, p=.163)$. Furthermore, significant interaction effect was found $(F(8,216)=6.707, p$ $\left.=.001, \eta^{2}=0.199\right)$, with three cueing contingencies differing in the first $\left(F(2,54)=5.04, p=.010, \eta^{2}=0.157\right)$ and fifth $(F$ $\left.(2,54)=6.15, p=.004, \eta^{2}=0.186\right)$ quintiles. For the trials of the first quintile, the search RTs in the LTM-matched condition was significantly shorter than those in the WM-matched condition $(p=.016)$. However, there were no significant differences when compared the neutral condition with both the LTM-matched and WM-matched conditions in these fastest trials. For the trials of the fifth quintile, the search RTs in the WM-matched condition were significantly shorter than those in the neutral $(p=.006)$ and LTM-matched $(p=.027)$ conditions.

Consistent with Experiment 1, the analysis of RTs from the change detection task showed a significant main effect of cueing contingency $\left(F(2,54)=18.836, p<.001, \eta^{2}=0.411\right)$. Participants were faster in the WM-matched condition as compared with the neutral and LTM-matched conditions (both $p s$ $<.001)$. Moreover, change detection accuracy $(F(2,54)=$ 14.192, $\left.p<.001, \eta^{2}=0.345\right)$ also demonstrated a similar pattern that the WM-matched condition was more accurate than the neutral and LTM-matched conditions (both $p s<$ $.001)$.
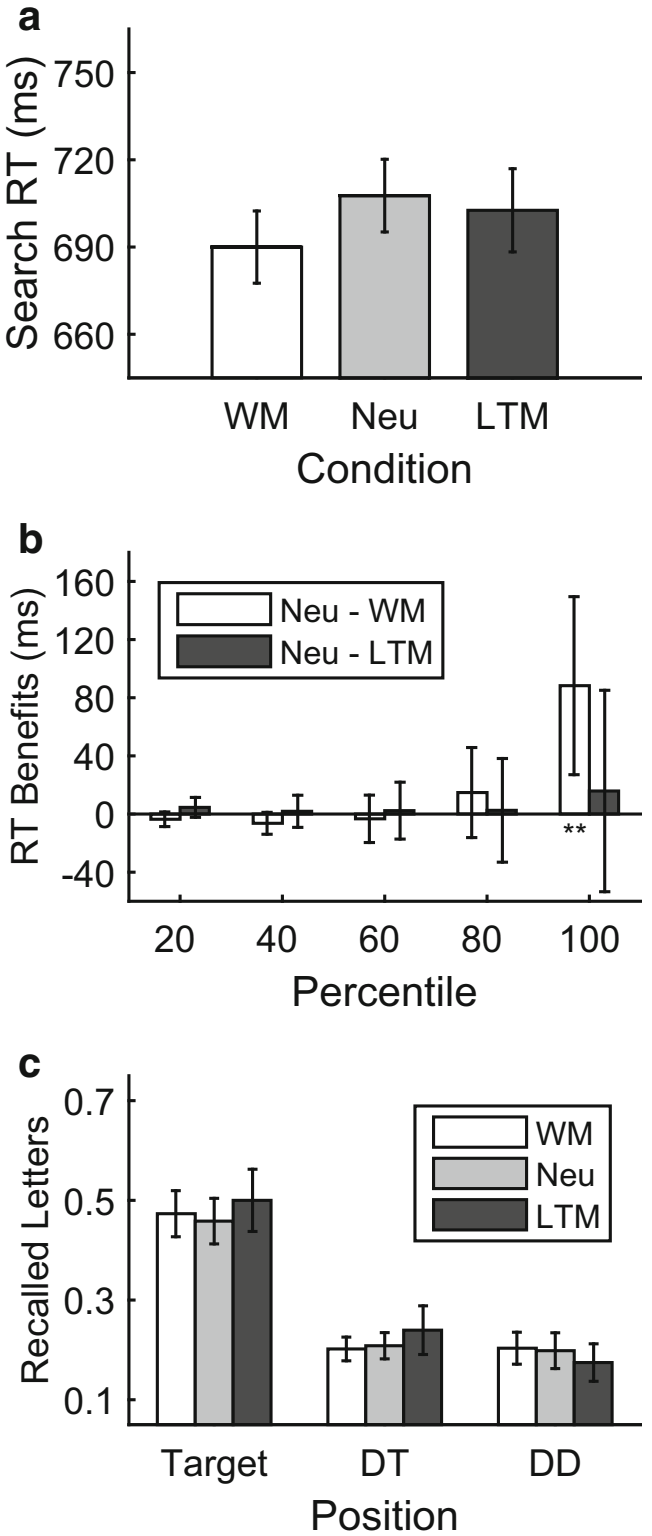

Fig. 3 Results of Experiment 2. (a) Search RTs of the three cueing contingencies in the search trials. (b) RT benefits in the WM-matched and LTM-matched conditions in five RT percentiles in the search trials. (c) Number of correctly recalled letters per location of each type (target, DT, and DD) in the probe trials. Error bars represent within-subject $95 \%$ confidence interval. Asterisks represent significances $(* p<.05, * * p<.01$, $* * * p<.001)$. $D T$ distractors in target color, $D D$ distractors in distractor color

\section{Probe trials}

Participants correctly recalled 1.49 letters per trial on average and there was no significant difference among three cueing contingencies $(F(2,54)=0.332, p=.719)$. The numbers of the correctly recalled letters per location of each type under different cueing contingencies were shown in Fig. 3c. A two-way repeated measures ANOVA (letter position $\times$ cueing 
contingency) revealed a significant main effect of letter position $\left(F(2,54)=91.432, p<.001, \eta^{2}=.772\right)$, but not of cueing contingency $(F(2,54)=1.989, p=.147)$ and their interaction $(F(4,108)=1.627, p=.188)$. Participants recalled more letters at the position of the target than at the positions of DT and DD (both $p s<.001$ ). Although change detection RTs of probe trials were roughly the same for the three cueing contingencies $(F(2,54)=0.936, p=0.378)$, a significant difference in change detection accuracy was observed $(F(2,54)=$ $\left.11.197, p<.001, \eta^{2}=0.293\right)$. The memory accuracy was higher in the WM-matched condition than that in the neutral $(p<.001)$ and LTM-matched $(p=.002)$ conditions.

\section{Discussion}

We did not find a significant overall cueing effect in search RTs in the varied cue condition, which was consistent with the findings from previous literature (Cunningham \& Egeth, 2016; Kerzel \& Barras, 2016; Noonan et al., 2016). However, when we examined the benefits of the cues in different RT quintiles, significant RT benefits in the WM-matched condition emerged in the trials with the longest RTs. These results agreed with the proposal that the suppression of cued distractors required sufficient cognitive control. The suppressive process required frequent updates of the cueing information in WM and only those trials with sufficiently long RTs to accomplish cognitive control in creating and executing rejection templates showed significant RT benefits. In contrast, we did not observe significant RT benefits in the LTM-matched condition, even among the trials with the longest RTs. One possible reason for the lack of RT benefits in the LTM-matched condition could be a weak LTM representation of the constant color, which will be addressed in Experiment 3 by adding a prior consolidation session. An alternative explanation was the failure in reactivating LTM information into WM when the distractors in the constant color appeared. A recent study has suggested that information retrieved from LTM was first represented in WM and therefore faced with the similar resource limitation (Fukuda \& Woodman, 2017). Since the switching of WM representations between trials dominated the utilization of cognitive control, the resources that were available for activating the LTM information for suppressive purpose could be greatly reduced in the varied cue condition.

In the probe trials, when the color cue changed every trial, neither a capture nor a suppression effect was found in the positions in the distractor color. Since we adopted a staircase procedure to control for the possibility that participants might resample the stimuli in search array to optimize performance in recall phase, we expected that no capture effect was observed in Experiment 2 at the positions of the distractors in the cued color. On the other hand, the results of the search trials indicated that the significant suppression effect was not evident across all trials but instead only evident in the trials with the longer RTs. This might provide the potential explanation of the absence of the suppression effect in the probe trials. Given the small proportion $(10 \%)$ of the probe trials, we could not split them into five RT quintiles.

\section{Experiment 3}

In Experiment 3, we aimed to investigate the suppression effect while placing the search items in a larger visual space $\left(12^{\circ} \times 12^{\circ}\right.$ vs. $4^{\circ} \times 4^{\circ}$ in Experiments 1 and 2) (e.g., Han \& Kim, 2009; Soto et al., 2005; Woodman \& Luck, 2007). This manipulation led to a significant increase in the spacing between search items, thus reducing the possibility of within receptive field competition among them (Desimone \& Duncan, 1995). With this change, we were able to control the possible contributions from the between items competition.

\section{Method}

\section{Participants}

Twenty-eight new participants (six males; age range: 18-27 years; mean age: 21.3 years; one left-handed) participated in the experiment and were paid for their participation. All participants had normal or corrected-to-normal visual acuity and normal color vision, none of them had known neurological or visual disorders.

\section{Stimuli and apparatus}

The stimuli and apparatus were the same as in Experiment 2, except that we replaced the search items with Landolt-squares $\left(0.6^{\circ} \times 0.6^{\circ}\right)$ in a larger visual space and used only six colors in this experiment.

\section{Procedure}

At the beginning of each trial, after the 1-s blank, a search array containing six Landolt- squares with different gap $\left(0.12^{\circ} \times 0.12^{\circ}\right)$ orientations was presented. Distractors in the search array had a gap either on the left or right while the only target had an upward or downward gap. The six search items formed an imaginary circle with a distance of $6^{\circ}$ to the center. Participants were instructed to find the target and indicate the location of the gap by pressing number 8 (upward gap) or 0 (downward gap) using their right hands. Similar to Experiment 2, we adopted a staircase procedure to obtain an appropriate presentation duration of the search array for each participants. We relaxed the criterion of threshold to $500 \mathrm{~ms}$ since the task was more difficult than that in Experiment 2. Participants' thresholds ranged from $115 \mathrm{~ms}$ to $414 \mathrm{~ms}$ with a 
mean of $280 \mathrm{~ms}$. Considering that the thresholds were long enough for participants to make saccades, we recorded eye movement using the Eyelink 1000 Plus $(1,000 \mathrm{~Hz}$, monocular) eye-tracker. Since maintaining varied WM representations increased WM load and therefore hampered reactivation of LTM, a consolidation session with 100 trials was conducted before the main experiment. In the consolidation session, no cue was presented before the onset of search display. There were only two conditions: neutral (the constant color did not appear in search array) and constant (the constant color was the distractor color). There were no probe trials in Experiment 3.

\section{Data analysis}

The procedures for data exclusion and statistical analysis were the same as in Experiment 1, resulting in 30.30\% of the trials being dropped for incorrect performance in either the search or change detection tasks. Furthermore, $2.20 \%$ and $4.06 \%$ of all search trials were excluded because of the out of RT range in the search and change detection tasks respectively.

\section{Results}

Data analysis was conducted by including and excluding the trials with eye movements and both procedures showed similar patterns of result. Hence, we reported the results including the trials with eye movements. We observed significant main effect of cueing contingency on neither search RTs $(F(2,54)=$ $0.681, p=.462$, Fig. 4a) nor accuracy $(F(2,54)=1.403, p=$ $.255)$. As shown in Fig. $4 \mathrm{~b}$, there was a significant main effect of RT quintile $\left(F(4,108)=220.957, p<.001, \eta^{2}=.891\right)$, as well as a marginal significant interaction $(F(8,216)=2.919, p$ $\left.=.051, \eta^{2}=0.098\right)$, but not of cueing contingency $(F(2,54)=$ $0.680, p=.463)$. The search RTs in the WM-matched condition were significantly shorter than in the neutral condition $(p$ $=.040)$ in the trials with the longest RTs. Additionally, there were significant main effects on change detection RTs $(F(2$, $\left.54)=8.797, p<.001, \eta^{2}=0.246\right)$ and accuracy $(F(2,54)=$ $\left.13.835, p<.001, \eta^{2}=0.339\right)$. Change detection RTs were slowest in the LTM-matched condition as compared with those in the WM-matched $(p=.001)$ and neutral $(p=.033)$ conditions. Change detection accuracy in the WM-matched condition was higher than those in the neutral and LTM-matched conditions (all $p s<=.001$ ).

\section{Discussion}

We replicated the findings in Experiment 2 by showing that the facilitation effect of the WM-matched condition was evident in the trials with the longest RTs. Additionally, we added a consolidation session to exclude the possibility that the null effect of the LTM-matched condition in Experiment 2 was due to participants' weak memory representation of the constant

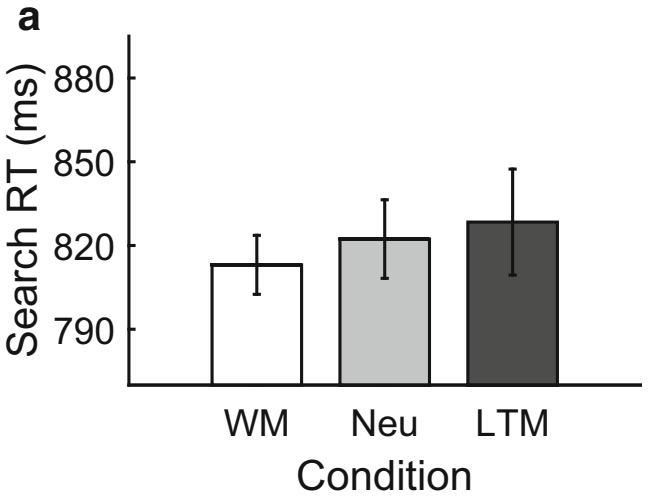

b

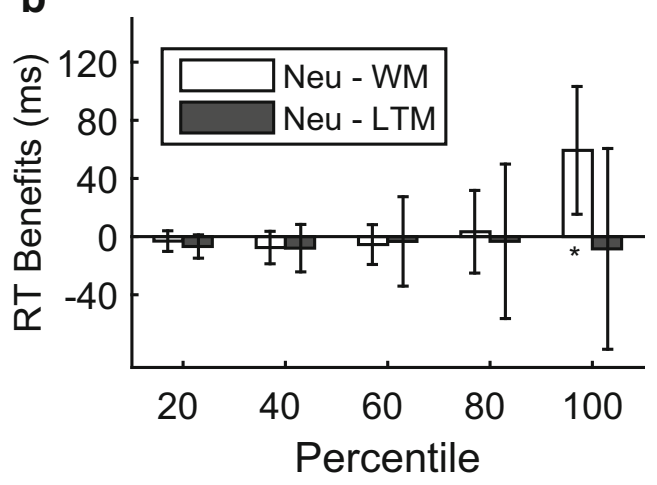

Fig. 4 Results of Experiment 3. (a) Search RTs of the three cueing contingencies. (b) RT benefits in the WM-matched and LTM-matched conditions in five RT percentiles. Error bars represent within-subject 95\% confidence interval. Asterisks represent significances $(* p<.05, * * p<.01$, $* * * p<.001)$

color. Lack of the RT benefits in the LTM-matched condition suggests that strong LTM representation might not be the critical factor that influences the LTM-based distractor suppression.

\section{General discussion}

WM plays an important role in maintaining and manipulating information for ongoing tasks (Baddeley, 1992; D'Esposito \& Postle, 2015). The present study focused on the top-down guidance from WM and LTM in distractor suppression by adopting a dual task paradigm with a to-be-ignored color cue. The results of Experiment 1 showed that both the WM and LTM representations under the fixed cue condition could effectively guide attention away from matching distractors. These facilitation effects were evident across the different quintiles of RT distribution. In Experiments 2 and 3, when the color cue varied from trial to trial, RT benefits of the WM-matched distractors occurred only in the trials with the longest RTs where sufficient cognitive control could be established. Nevertheless, participants could not take the advantage of the LTM representation in the varied cue condition, 
even among the trials with the longest RTs. These results suggest that the availability of cognitive control is a critical factor for effective WM-guided distractor suppression, whereas the LTM-guided suppression occurs only if sufficient resource of WM is accessible by LTM reactivation.

As a result of the brain's evolutionary development, stimulus signaling physical or valence-based significance could distract ongoing process of attentional allocation and may interfere with the task at hand (Anderson, Laurent, \& Yantis, 2011; Schmidt, Belopolsky, \& Theeuwes, 2015; Theeuwes, 1992). Recent studies have further suggested that task-irrelevant distractors that matched with the internally held WM representations could also automatically capture attention and impair task performance (Olivers et al., 2006; Soto et al., 2005; Mannan et al., 2010). However, other studies have pointed out that the influence of WM on attention deployment is rather flexible and could be strategically controlled (Dalvit \& Eimer, 2011; Arita et al., 2012; Han \& Kim, 2009; Woodman \& Luck, 2007). Our results agreed with the latter proposal by demonstrating that voluntarily using the to-be-ignored cue could overcome the attraction from WM-matched distractor. In particular, in Experiment 1, we showed similar results as the existing literature that the interference of WM-matched distractors could be reversed into facilitation when to-be-ignored distractor feature was fixed (Cunningham \& Egeth, 2016; Gaspelin et al., 2015), indicating the strategic development of a rejection template using the color cue. One might argue that the significant suppression effect found in Experiment 1 was caused by the absence of articulatory suppression task. However, Cunningham and Egeth (2016) demonstrated similar RT benefits of inhibiting non-targets in the fixed cue condition where no articulatory suppression task was used. Moreover, previous study has suggested that articulatory suppression task was not necessary to induce memory-driven attentional capture (Olivers, 2009) and even reduced the WM-driven capture effect if it was applied (Soto \& Humphreys, 2008). Therefore, RT benefits we observed in Experiment 1 could not be attributed to the lack of articulatory suppression task.

Flexible control of attentional allocation is resource consuming in front of a rapidly changing environment. Indeed, previous literature showed that distractor suppression effect disappeared in the varied cue condition (Cunningham \& Egeth, 2016; Kerzel \& Barras, 2016), suggesting a failure of forming a rejection template under such situation. However, in Experiments 2 and 3, we found RT benefits in the trials with the longest RTs, indicating that participants could form a rejection template based on the varied cues. This result was consistent with the findings from Han and Kim (2009) who compared the suppression effect between participants with different search speeds and showed RT benefits only in the slow group. Importantly, our results also demonstrated that the RT benefits in distractor suppression were evident across the participants with different levels of search speed.
The sustained maintenance of goal-relevant information before the onset of an event is considered an important component of proactive control (Braver, Gray \& Burgess, 2007; Braver, 2012). In the present study, the WM-matched conditions in the three experiments were specific instances of such proactive control. Under the fixed cue condition in Experiment 1 , repetitively suppressing the same WM-matched distractor required less effort and thus did not rely on large consumption of cognitive resources. Hence, the facilitation effect in the WM-matched condition occurred even in the trials with shortest RTs. However, under the varied cue condition in Experiments 2 and 3, participants needed to update the WM representations of the cues in every trial, leading to a greater occupation of the cognitive resources and longer RTs for the effective cue-induced cognitive control. Thus, we could observe a significant facilitation effect in the WM-matched condition only in the trials with the longest RTs.

In contrast to the WM-matched condition, we observed facilitation effect in the LTM-matched condition only in Experiment 1, where the WM-held cues were fixed within a block. Interestingly, under the fixed cue condition, the facilitation effect in the LTM-matched condition was comparable with that in WM-matched condition. There are two possible interpretations for observing the comparable facilitation effects between the two conditions. First, the representations of the fixed cues were transferred to LTM after a few repetitions in the block, and it was the LTM-held cues that facilitated the search performance. Second, the LTM information of the constant color was loaded into WM throughout the experiment given that human can remember approximately four colors in their WM (Luck \& Vogel, 1997). While both interpretations may account for the observed effects under the fixed cue condition, we consider the latter one as more plausible for the following three reasons. First, as suggested by both the classical theories (Atkinson \& Shiffrin, 1968; Baddeley \& Hitch, 1974) and the recent neurophysiological evidence (Fukuda \& Woodman, 2017), the information retrieved from LTM is first represented in WM. It was trivial for the participants to retrieve the LTM information routinely in every trial. Therefore, holding the color cue and the constant color in WM rather than in LTM could reduce the retrieval cost significantly. Second, the facilitation effect in both conditions occurred even in the trials with the shortest RTs, suggesting that participants were ready to suppress those memory-matched distractors before the onset of the search array. In particular, the search array was presented for $150 \mathrm{~ms}$ in Experiment 1, making the LTM-based reactive rejection mechanism unlikely to be fulfilled as this engage-disengage process requires at least $150-300 \mathrm{~ms}$ to complete in the presence of the search display (Geng, 2014; Moher \& Egeth, 2012; Theeuwes, 2010). Third, recent studies have shown that multiple WM representations could control attention simultaneously (Chen \& Du, 2017; Hollingworth \& Beck, 2016). Therefore, it was functionally possible for the 
participants to maintain two colors in WM (i.e., the cueing color and the constant color) when rapid switching was not required in Experiment 1. In Experiments 2 and 3, reactive control with late correction mechanism was more likely to play a role in the LTM-matched condition when the to-be-ignore cues varied on a trial-by-trial basis (Geng, 2014). In the present study, the appearance of the LTM-matched distractors was less prevalent and unpredictable. Rapid switch of the WM representations taxed a significant amount of cognitive capacity, thus making holding the constant color in WM a less efficient approach for performing the task. Taken together, our results suggested that facilitation of distractor suppression by LTM representations also required sufficient availability of cognitive control. However, the WM-LTM interaction in visual search is a complex process. Future investigations, particularly combined with neuroimaging and neurophysiological recordings, are required to elucidate its underlying mechanisms.

To conclude, our study showed that WM representation could be voluntarily used to suppress its associated distractors in the fixed cue condition. When varying WM-held distractor cues from trial to trial, WM-guided suppression occurred only in the trials with sufficient processing time that allowed successful creation and utilization of a rejection template. On the other hand, the LTM representation of the to-be-ignored feature could facilitate search performance only in the fixed cue condition in which sufficient WM resource is accessible by LTM reactivation. Future investigations with neuroimaging techniques are required to explore the neural mechanisms underlying these behavioral phenomena.

Acknowledgements This work was supported by the National Natural Science Foundation of China $(31470974,31230029)$ and the National Key R\&D Program of China (2017YFB1002503).

\section{References}

Anderson, B. A., Laurent, P. A., \& Yantis, S. (2011). Value-driven attentional capture. Proceedings of the National Academy of Sciences, 108(25), 10367-10371.

Arita, J. T., Carlisle, N. B., \& Woodman, G. F. (2012). Templates for rejection: Configuring attention to ignore task-irrelevant features. Journal of experimental psychology: human perception and performance, 38(3), 580-584.

Atkinson, R. C., \& Shiffrin, R. M. (1968). Human memory: A proposed system and its control processes. Psychology of learning and motivation, 2, 89-195.

Baddeley, A. (1992). Working memory. Science, 255(5044), 556-559.

Baddeley, A. D., \& Hitch, G. (1974). Working memory. Psychology of learning and motivation, 8, 47-89.

Brainard, D. H. (1997). The psychophysics toolbox. Spatial Vision, 10(4), 433-436.

Braver, T. S. (2012). The variable nature of cognitive control: A dual mechanisms framework. Trends in cognitive sciences, 16(2), 106113.

Braver, T. S., Gray, J. R., \& Burgess, G. C. (2007). Explaining the many varieties of working memory variation: Dual mechanisms of cognitive control. In Variation in Working Memory (Conway, A. et al., eds), pp. 76-106, New York, NY: Oxford University Press.

Bundesen, C. (1990). A theory of visual attention. Psychological review, 97(4), 523.

Carlisle, N. B., \& Woodman, G. F. (2011). When memory is not enough: Electrophysiological evidence for goal-dependent use of working memory representations in guiding visual attention. Journal of Cognitive Neuroscience, 23(10), 2650-2664.

Chen, Y., \& Du, F. (2017). Two visual working memory representations simultaneously control attention. Scientific reports, 7, 6107.

Cunningham, C. A., \& Egeth, H. E. (2016). Taming the white bear initial costs and eventual benefits of distractor inhibition. Psychological science, 27(4), 476-485.

Dalvit, S., \& Eimer, M. (2011). Memory-driven attentional capture is modulated by temporal task demands. Visual Cognition, 19(2), $145-153$.

Desimone, R., \& Duncan, J. (1995). Neural mechanisms of selective visual attention. Annual review of neuroscience, 18(1), 193-222.

D'esposito, M., \& Postle, B. R. (2015). The cognitive neuroscience of working memory. Annual review of psychology, 66, 115-142.

Downing, P., \& Dodds, C. (2004). Competition in visual working memory for control of search. Visual Cognition, 11(6), 689-703.

Downing, P. E. (2000). Interactions between visual working memory and selective attention. Psychological Science, 11(6), 467-473.

Fan, J. E., \& Turk-Browne, N. B. (2016). Incidental biasing of attention from visual long-term memory. Journal of Experimental Psychology: Learning, Memory, and Cognition, 42(6), 970.

Faul, F., Erdfelder, E., Lang, A. G., \& Buchner, A. (2007). G* Power 3: A flexible statistical power analysis program for the social, behavioral, and biomedical sciences. Behavior research methods, 39(2), 175191.

Fukuda, K., \& Woodman, G. F. (2017). Visual working memory buffers information retrieved from visual longterm memory. Proceedings of the National Academy of Sciences, 114, 5306-5311.

Gaspelin, N., Leonard, C. J., \& Luck, S. J. (2015). Direct evidence for active suppression of salient-butirrelevant sensory inputs. Psychological science, 26(11), 1740-1750.

Gaspelin, N., Leonard, C. J., \& Luck, S. J. (2017). Suppression of overt attentional capture by salient-but-irrelevant color singletons. Attention, Perception, \& Psychophysics, 79(1), 45-62.

Geng, J. J. (2014). Attentional mechanisms of distractor suppression. Current Directions in Psychological Science, 23(2), 147-153.

Gong, M., Jia, K., \& Li, S. (2017). Perceptual competition promotes suppression of reward salience in behavioral selection and neural representation. Journal of Neuroscience, 37(26), 6242-6252.

Gong, M., Yang, F., \& Li, S. (2016). Reward association facilitates distractor suppression in human visual search. European Journal of Neuroscience, 43(7), 942-953.

Han, S. W., \& Kim, M. S. (2009). Do the contents of working memory capture attention? Yes, but cognitive control matters. Journal of Experimental Psychology: Human Perception and Performance, 35(5), 1292.

Hollingworth, A., \& Beck, V. M. (2016). Memory-based attention capture when multiple items are maintained in visual working memory. Journal of experimental psychology: human perception and performance, 42(7), 911.

Houtkamp, R., \& Roelfsema, P. R. (2006). The effect of items in working memory on the deployment of attention and the eyes during visual search. Journal of Experimental Psychology: Human Perception and Performance, 32(2), 423.

Hutchinson, J. B., \& Turk-Browne, N. B. (2012). Memory-guided attention: Control from multiple memory systems. Trends in cognitive sciences, 16(12), 576-579.

Kerzel, D., \& Barras, C. (2016). Distractor rejection in visual search breaks down with more than a single distractor feature. Journal of 
experimental psychology: human perception and performance, 42(5), 648

Kiyonaga, A., Egner, T., \& Soto, D. (2012). Cognitive control over working memory biases of selection. Psychonomic bulletin \& review, 19(4), 639-646.

Kleiner, M., Brainard, D., Pelli, D., Ingling, A., Murray, R., \& Broussard, C. (2007). What's new in Psychtoolbox-3. Perception, 36(14), 1.

Lu, J., Tian, L., Zhang, J., Wang, J., Ye, C., \& Liu, Q. (2017). Strategic inhibition of distractors with visual working memory contents after involuntary attention capture. Scientific reports, 7(1), 16314.

Luck, S. J., \& Vogel, E. K. (1997). The capacity of visual working memory for features and conjunctions. Nature, 390(6657), 279.

Mannan, S. K., Kennard, C., Potter, D., Pan, Y., \& Soto, D. (2010). Early oculomotor capture by new onsets driven by the contents of working memory. Vision research, 50(16), 1590-1597.

Moher, J., \& Egeth, H. E. (2012). The ignoring paradox: Cueing distractor features leads first to selection, then to inhibition of tobe-ignored items. Attention, Perception, \& Psychophysics, 74(8), $1590-1605$

Moher, J., Lakshmanan, B. M., Egeth, H. E., \& Ewen, J. B. (2014). Inhibition drives early feature-based attention. Psychological science, 25(2), 315-324.

Noonan, M. P., Adamian, N., Pike, A., Printzlau, F., Crittenden, B. M., \& Stokes, M. G. (2016). Distinct mechanisms for distractor suppression and target facilitation. Journal of Neuroscience, 36(6), 17971807.

Olivers, C. N. (2009). What drives memory-driven attentional capture? The effects of memory type, display type, and search type. Journal of Experimental Psychology: Human Perception and Performance, 35(5), 1275.

Olivers, C. N. (2011). Long-term visual associations affect attentional guidance. Acta psychologica, 137(2), 243-247.

Olivers, C. N., Meijer, F., \& Theeuwes, J. (2006). Feature-based memorydriven attentional capture: Visual working memory content affects visual attention. Journal of Experimental Psychology: Human Perception and Performance, 32(5), 1243.

Olivers, C. N., Peters, J., Houtkamp, R., \& Roelfsema, P. R. (2011). Different states in visual working memory: When it guides attention and when it does not. Trends in cognitive sciences, 15(7), 327-334.

Peters, J. C., Goebel, R., \& Roelfsema, P. R. (2009). Remembered but unused: The accessory items in working memory that do not guide attention. Journal of Cognitive Neuroscience, 21(6), 1081-1091.

Schmidt, L. J., Belopolsky, A. V., \& Theeuwes, J. (2015). Attentional capture by signals of threat. Cognition and emotion, 29(4), 687-694.
Soto, D., Heinke, D., Humphreys, G. W., \& Blanco, M. J. (2005). Early, involuntary top-down guidance of attention from working memory. Journal of Experimental Psychology: Human Perception and Performance, 31(2), 248.

Soto, D., Hodsoll, J., Rotshtein, P., \& Humphreys, G. W. (2008). Automatic guidance of attention from working memory. Trends in cognitive sciences, 12(9), 342-348.

Soto, D., \& Humphreys, G. W. (2007). Automatic guidance of visual attention from verbal working memory. Journal of Experimental Psychology Human Perception and Performance, 33(3), 730.

Soto, D., \& Humphreys, G. W. (2008). Stressing the mind: The effect of cognitive load and articulatory suppression on attentional guidance from working memory. Attention, Perception, \& Psychophysics, 70(5), 924-934.

Soto, D., \& Humphreys, G. W. (2009). Automatic selection of irrelevant object features through working memory: Evidence for top-down attentional capture. Experimental psychology, 56(3), 165-172.

Stokes, M. G., Atherton, K., Patai, E. Z., \& Nobre, A. C. (2012). Longterm memory prepares neural activity for perception. Proceedings of the National Academy of Sciences, 109(6), E360-E367.

Theeuwes, J. (1992). Perceptual selectivity for color and form. Attention, Perception, \& Psychophysics, 51(6), 599-606.

Theeuwes, J. (2010). Top-down and bottom-up control of visual selection. Acta Psychologica, 135(2), 77-99.

Van Selst, M., \& Jolicoeur, P. (1994). A solution to the effect of sample size on outlier elimination. Quarterly Journal of Experimental Psychology, 47A, 631-650.

Vatterott, D. B., \& Vecera, S. P. (2012). Experience-dependent attentional tuning of distractor rejection. Psychonomic bulletin \& review, 19(5), 871-878.

Wolfe, J. M. (1994). Guided search 2.0 a revised model of visual search. Psychonomic bulletin \& review, 1(2), 202-238.

Woodman, G. F., Carlisle, N. B., \& Reinhart, R. M. (2013). Where do we store the memory representations that guide attention? Journal of Vision, 13(3), 1-1.

Woodman, G. F., \& Chun, M. M. (2006). The role of working memory and long-term memory in visual search. Visual Cognition, 14(4-8), 808-830.

Woodman, G. F., \& Luck, S. J. (2007). Do the contents of visual working memory automatically influence attentional selection during visual search? Journal of Experimental Psychology: Human Perception and Performance, 33(2), 363.

Yi, D. J., Turk-Browne, N. B., Chun, M. M., \& Johnson, M. K. (2008). When a thought equals a look: Refreshing enhances perceptual memory. Journal of Cognitive Neuroscience, 20(8), 1371-1380. 